\title{
Simulation Analysis of Static Recrystallization by Molecular Automaton
}

\author{
Yi HE, YiPing XU \\ LuoYang Institute of Science and Technology, Luoyang 471023, China \\ Lit_xyp@lit.edu.cn
}

Keywords: Molecular automaton, static recrystallization, simulation, analysis

\begin{abstract}
In this paper, people mainly take three two-dimensional arrays State, Energy and Ori (Ori is the positive integer of $1 \leq$ Ori $\leq$ Or $i_{\max }$ ) to perform the molecular states and build models. According to the simulation results of molecular automaton, people have a research on the change and distribution of the grain diameter and the number of side during the process of static recrystallization, and conclude as follows, the relationship between the disappearing rate of individual deformation grain and the growth rate of individual recrystallization grain is characterized by randomness during the whole recrystallization process; the more the deformation, the smaller the mean diameter of grain is, at the finished moment of recrystallization; the grain diameters are not homogeneous during the static recrystallization process and at the finished moment of static recrystallization.
\end{abstract}

\section{Foreword}

In recent years, it has been the hot spot of the research to simulate the microstructure evolution by molecular automaton. Since Hesselbarth ${ }^{[1]}$ firstly simulated the recrystallization in two dimensional condition by molecular automaton in 1991, the molecular automaton model of recrystallization has been developed rapidly. However, the effect of the plastic deformation before the static recrystallization on grain forms has not been considered in the former models, and in fact, certain deformation is the essential requirement of the static recrystallization. Therefore, based on the parent phase structure of the equiaxed grains, the author can get the corresponding deformation structure through mesh mapping. On the basis of the above research, the author can build the static recrystallization model, lay emphasis on the analysis of the change rule of grain sizes, the distribution of grains diameter and the number of sides, and make a further understanding on the static recrystallization process.

\section{The building of molecular automaton model}

In this paper, people mainly take three two-dimensional arrays State, Energy and Ori (Ori is the positive integer of $1 \leq$ Ori $\leq$ Ori $\left.i_{\max }\right)$ to illustrate the molecular states in the research models. State can record whether the molecule can be recrystallized, and the result " 0 " means the molecule has a deformed structure, "1" means the molecule forms the recrystallization core, and " 2 " means the molecule can form recrystallization through core growth. Energy records the storage energy of molecule, which can be taken as the driving force of molecular recrystallization. Ori illustrates the molecular motion orientation. The molecules with the same orientation can form a grain, while the grains with different orientation can form natural grain boundary. The initial grain orientation value Ori can be obtained after the different effects of the equiaxed grains deformation $e$. During the recrystallization process, with the extending of the time step, the deformed grains can be swallowed by the new-formed recrystallization core, and at the same time, three state variables of the molecule has the corresponding change and the change is stored by the computer. After the simulation, people can make an analysis on the whole structure evolution according to the change of the state variables. 


\section{Simulation results and analysis}

Considering the small simulation area, people can propose the temperature distribution is even, $T=$ $1273 \mathrm{~K}$, and the temperature remains unchanged in the whole static recrystallization process. The sample size is $a \times b=200 \times 200 \mu \mathrm{m}^{2}$, which is obtained after the deformation when the equiaxed parent phase structure can produce the deformation $e=0.25,0.5,0.75$, and the grains diameters of the parent phase structure, before the deformation with different effects, are all the same, $\mathrm{D}_{0}=20 \mu \mathrm{m}$.

\section{The change of grain diameter}

The diameter change of the individual grain. In order to research the change of the individual grain diameter in the static recrystallization process, when the deformation in the static recrystallization simulation requires $e=0.5$, people can choose 5 deformed grains in random, recording as grain 1 to grain 5 separately, and the other 5 recrystallized grains are recorded as grain 6 to grain 10 separately. People should follow the diameter change of the grains, as shown in Fig 1. Seen from Fig 1, all the deformed grains are disappeared after some time steps, but the big size grains are not disappeared slowly and the small size are not disappeared rapidly. The disappearing speed of a individual grain is in random, and there in no rule for the change. The growth speeds of the new-formed grains are various, and there is not complete relevance between the final grain diameter and the core forming time, early or late, because the surrounding recrystallized grains affect the core growth. If one core is developed earlier, and there are many recrystallized grains in it's surrounding, in the growing process, the core can collide with the surrounding recrystallized grains, then the core will stop grow, so the grain diameter will be small. Thus, only meet two requirements, one is core forming relative earlier, and the other is that there is no collision with other new recrystallized grains, can the new grain be relative big. The grain meeting the above conditions is called a grain with a prior growing condition.
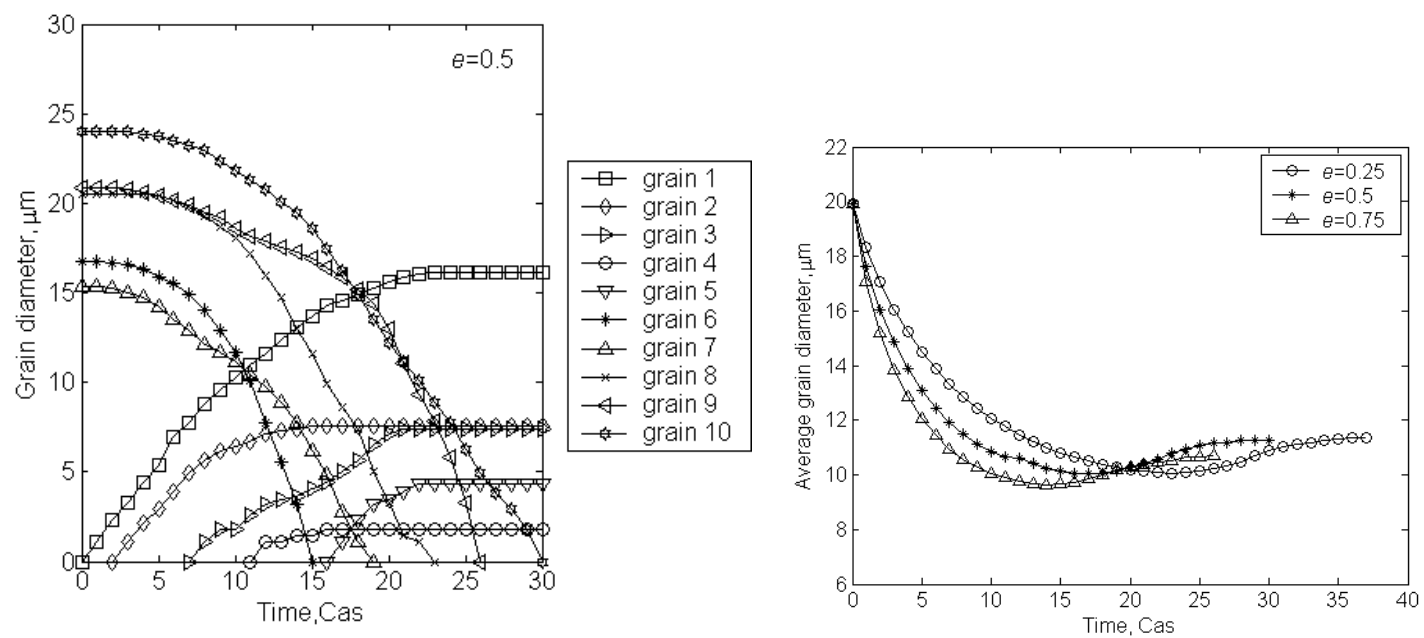

Fig 1 Individual grain diameter changed with time

Fig 2 Average grain diameter changed with time

The change of the average grain diameter. Although there is no obvious change rule of the individual grain diameter in the recrystallization process, there are some rules in the whole change of the average grain diameter. Fig 2 has shown the average diameter change along with time in various deformation effects. As shown from it, during the long period of time after the beginning of recrystallization, the many recrystallized cores are formed with the time extended, and the swallowed speed of original grain is relatively low, which cause the number of the grains in the whole simulated area increasingly rises up, and the average grain diameter become short. When the recrystallization reaches certain degree, the new-deformed core number and the swallowed number of the original grains are almost the same, and the average diameter is with little change. Along with the recrystallization, the place for the growing new cores become small, and the new-formed grains become less, even less than the swallowed original deformed grains, which causing the average grain diameter become a bit of longer just in the period before the complete of recrystallization. Comparing the grain diameter change in various deformation effects, people can know that the more the 
deformation is, the less the average diameter will be, and if the less the minimum of the grain diameter is, the less the final obtained grain diameter is. Usually, people can not exactly test the moment when the recrystallization is just complete in the experiment, so the recrystallization structure people observed in the experiment belongs to the structure in the recrystallization process. Experimental results demonstrate that the average grain diameter become smaller along with the increasing of the deformation effect in the recrystallization process. This simulation result accords well with the experimental result.

The distribution of grain diameter and the number of side.

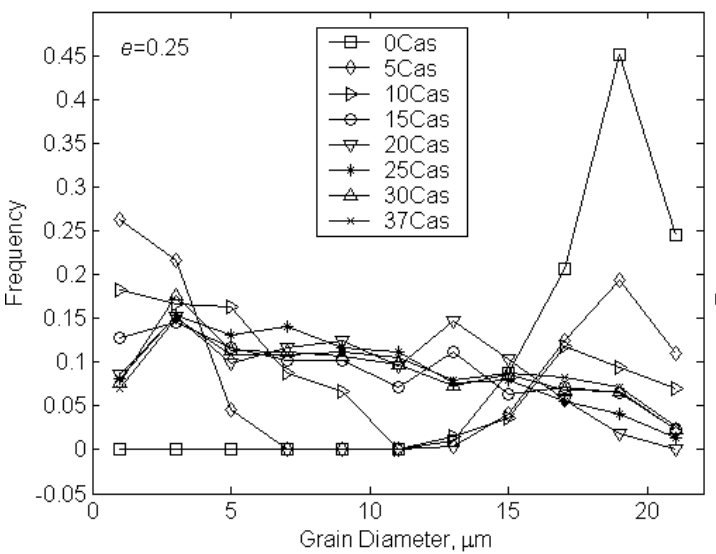

(a)

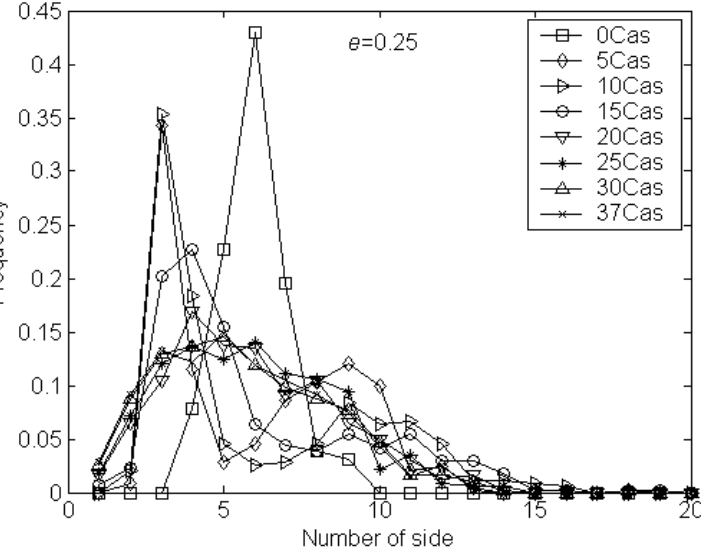

(b)

Fig 3 Distribution of grain diameter and the number of side

(a) distribution of grain diameter (b) distribution of the number of side

Fig 3 shows the distribution of grain diameter and the number of side in the simulation when $e=0.25$. Seen from Fig 3 (a), the grain diameter of the initial equiaxed grain structure is basically distributed between $15 \mu \mathrm{m}$ and $20 \mu \mathrm{m}$, and the grain size is moderate. During the beginning period of the recrystallization $(0 \sim 5 \mathrm{Cas}$, shift amount $<5 \%)$, the small size grains $(<5 \mu \mathrm{m})$ greatly increase, with a rather high frequency, because there are many new formed cores and the core can not develop to a big one at the beginning. Along with the development of the recrystallization (5 20Cas), the new core become big by swallowing the surrounding deformed structure, and the original deformed grain is swallowed, so the diameter become small. In the above conditions, the occupied frequency of the small size grain decreases, and the occupied frequency of the moderate size grain $(5 \sim 15 \mu \mathrm{m})$ increases. Seen from the change of the big size grain $(>15 \mu \mathrm{m})$, the occupied frequency of the big size gradually decreases, even to 0 . During the late period of recrystallization (20 37Cas, shift amount $>85 \%$ ), the new-formed cores are relatively less, and the swallowed grains in each time step are less. The distribution of the grain diameter is in a stable trend, without a obvious change. One thing to be mentioned is that there are few cores in prior growing condition can develop into big size grain in the late period of recrystallization, and the big size grain frequency increases, which is one of the reasons for the uneven distribution of the recrystallized grain size. The other reason is that some recrystallized grain core directly contacts with the surrounding recrystallized grains before it grow well, which make the small size grain keep the small diameter till the end of the recrystallization. Seen from the experiment, when the recrystallization is finished, the frequency of big and small size grains is lower than 0.1 , and super size grain is not formed in the recrystallization process, which ensures the abnormal big size grain will not appear in the later developing process of the growing grain.

Seen from the Fig 3 (b), people can find the change rule of the number of side directly. In the initial time, the number of side is distributed around 4 9, and the rate of the grain whose distribution around $5 \sim 7$ is up to $80 \%$ or more. During the beginning period of the recrystallization, the cores are mostly formed in triangular grain boundaries junction, and the new-formed cores and the small size grains are all contiguous with three original deformed grains, which cause the high frequency of the three-side grain. Along with the development of the recrystallization, the original deformed grains disappear, and the new grains can contact with each other, so the frequency of the grains with fewer 
sides gradually decreases. Many new grains are formed around some original deformed grains, which increases the number of side, even up to 15 sides in certain moment. During the late period of the recrystallization, with the disappearing of the grains with abnormal many sides, the new grains begin to contact with each other. At the finished moment of recrystallization, the distribution of the grain diameter is uneven. Some grain cores with the prior growing conditions can develop into the big grains, and there are many small grains around them, which make the big grains with many sides, while small grains with much less sides, so the distribution of the number of side is uneven. However, the proportions the grains with few sides and many sides have taken are not high enough. The grains with $4 \sim 10$ sides have taken the proportion up to $80 \%$, and the distribution of the number of side belongs to a normal distribution, which agree with the basic rules of the statistics.

\section{Conclusion}

In the process of static recrystallization, it shows randomness on the research of the disappearing speed of the individual deformed grain and the growing speed of the individual recrystallized grain. Only meet the following two requirements can the core develops into the big grain: 1) the core is formed early; 2) without contacting with other new grains around it in the early period of the growing process.

The simulation results show that, during the long period after the beginning of the static recrystallization, the average grain diameter gradually decreases along with time extending, and the more the deformation effects are, the less the average grain diameter is. When the recrystallization reaches a certain degree, the quantity of the new-deformed cores is equivalent to the quantity of the swallowed original grains, and the average grain diameter comes to stable. Along with the further development of the recrystallization, the average diameter shows little increasing instead. The more the deformation effects are, the less the average grain diameter is at the finished moment of the recrystallization. The simulation result agrees well with the experimental result.

The simulation results show that, the grain diameter is uneven, both in the static recrystallization process and at the finished moment of the recrystallization, respectively, but the total frequency occupancy rate of the big size and small size grains is lower than 0.1 , and the super size grains are not formed during the recrystallization. Usually, the big size grain has much more sides, and the small size grain has less sides. The grains with less sides and much more sides do not have a high occupancy rate, and the grains with $4 \sim 10$ sides have a high rate up to $80 \%$. The distribution of the number of side belongs to a positive normal distribution, which agree with the basic rules of statistics.

\section{Reference}

[1] Hesselbarth H W, Gobel I R. Simulation of Recrystallization by Cellular Automata[J]. Acta Metallurgica et Materialia. 1991, 39 (9): 2135-2143.

[2] Pezzee C F, Dunand D C. The Impingement Effect of an Inert Immobile Second Phase on he Recrystallization of a Matrix[J]. Acta Metallurgical et Materialia. 1994, 42 (5):1509-1524.

[3] Davies C H J. The Effect of Neighbourhood on The Kinetics of a Cellular Automaton Recrystallisation Model[J]. Scripta Metallurgical et Materialia. 1995, 33 (7): 1139-1143.

[4] Davies C H J. Growth of Nuclei in a Cellular Automaton Simulation of Recrystallisation[J]. Scripta Materialia. 1997, 36 (1): 35-40.

[5] Davies C H J, Hong L. The Cellular Automaton Simulation of Static Recrystallisation in Cold-Rolled AA1050[J]. Scripta Materialia. 1999, 40 (10): 1145-1150.

[6] Goetz R L, Seetharaman V. Static Recrystallization Kinetics with Homogeneous and Heterogeneous Nucleation Using a Cellular Automata Model[J]. Metallurgical and MaterialsTransactions. 1998, 29A: 2307-2321. 
[7] Marx V, Reher F R, Gottstein G. Simulation of Primary Recrystallization Using a Modified Three- Dimensional Cellular Automaton[J]. Acta Materialia. 1999, 47 (4): 1219-1230.

[8] Gottstein G, Marx V, Sebald R. Integral recrystallization modeling[J]. Journal of Shanghai Jiaotong University. 2000, 5(1): 49 57.

[9] Gottstein G, Marx V, Sebald R. Integral recrystallization modeling: from cellular automata to finite element analysis[A]. The fouth International Conference on Recrystallization and Related Phnomena. Edited by Sakai T and Suzuki H G. The Japan Institute of Metals, Japan, 1999: $15 \sim 24$.

[10]Raabe D. Introduction of a Three-Dimensional Cellular Automaton with a Probabilistic Switching Rule for the Discrete Mesoscale Simulation of Recrystallization Phenomena[J]. Phil. Mag. 1999, 79 (10): 2339-2358. 